\title{
OPEN Spatial patterns of pathogen prevalence in questing Ixodes ricinus nymphs in southern Scandinavia, 2016
}

\author{
Lene Jung Kjær@1 ${ }^{1 凶}$, Kirstine Klitgaard ${ }^{2}$, Arnulf Soleng ${ }^{3}$, Kristin Skarsfjord Edgar ${ }^{3}$, \\ Heidi Elisabeth H. Lindstedt ${ }^{3}$, Katrine M. Paulsen ${ }^{4,5}$, Åshild Kristine Andreassen ${ }^{4}$, \\ Lars Korslund ${ }^{6}$, Vivian Kjelland ${ }^{6,7}$, Audun Slettan ${ }^{6}$, Snorre Stuen ${ }^{8}$, Petter Kjellander ${ }^{9}$, \\ Madeleine Christensson ${ }^{9}$, Malin Teräväinen ${ }^{9}$, Andreas Baum ${ }^{10}$, Laura Mark Jensen ${ }^{1}$ \& \\ René Bødker ${ }^{1}$
}

Tick-borne pathogens cause diseases in animals and humans, and tick-borne disease incidence is increasing in many parts of the world. There is a need to assess the distribution of tick-borne pathogens and identify potential risk areas. We collected 29,440 tick nymphs from 50 sites in Scandinavia from August to September, 2016. We tested ticks in a real-time PCR chip, screening for 19 vector-associated pathogens. We analysed spatial patterns, mapped the prevalence of each pathogen and used machine learning algorithms and environmental variables to develop predictive prevalence models. All 50 sites had a pool prevalence of at least $33 \%$ for one or more pathogens, the most prevalent being Borrelia afzelii, B. garinii, Rickettsia helvetica, Anaplasma phagocytophilum, and Neoehrlichia mikurensis. There were large differences in pathogen prevalence between sites, but we identified only limited geographical clustering. The prevalence models performed poorly, with only models for $R$. helvetica and $N$. mikurensis having moderate predictive power (normalized RMSE from $0.74-0.75, R^{2}$ from $0.43-0.48$ ). The poor performance of the majority of our prevalence models suggest that the used environmental and climatic variables alone do not explain pathogen prevalence patterns in Scandinavia, although previously the same variables successfully predicted spatial patterns of ticks in the same area.

Ticks are blood-sucking arthropods and transmit a wide range of disease-causing pathogens impacting both humans and animals ${ }^{1-3}$. They are capable of transmitting bacteria, viruses and protozoa, to their vertebrate hosts via blood meals, and the diseases caused by these etiological agents affect both animals and humans ${ }^{1,4}$. Tick-borne diseases have increased in incidence and geographical range over the last decades ${ }^{4-6}$ and previously, 30 European Ministries of Health have recognised vector-borne diseases as the "biggest threat arising from environmental change", with tick-borne encephalitis (TBE) and Lyme borreliosis (LB) ranking high on the list ${ }^{7,8}$. Predicted temperature increase, resulting from global warming ${ }^{9}$, could potentially cause tick range expansions, prolonged season of tick activity, changes to tick development rate and reproduction as well as the development rates of the pathogens these vectors may carry ${ }^{10-12}$. In Scandinavia, LB and TBE show an increasing trend, particularly in Norway and Sweden ${ }^{13-16}$, giving rise to public health concerns.

\footnotetext{
${ }^{1}$ Department of Veterinary and Animal Sciences, Faculty of Health and Medical Sciences, University of Copenhagen, Frederiksberg, Denmark. 'Department for Diagnostics and Scientific Advice, National Veterinary Institute, Technical University of Denmark, Lyngby, Denmark. ${ }^{3}$ Department of Pest Control, Norwegian Institute of Public Health, Oslo, Norway. ${ }^{4}$ Department of Virology, Norwegian Institute of Public Health, Oslo, Norway. ${ }^{5}$ Department of Production Animal Clinical Sciences, Norwegian University of Life Sciences, Oslo, Norway. ${ }^{6}$ Department of Natural Sciences, University of Agder, Kristiansand, Norway. ${ }^{7}$ Research Unit, Sørlandet Hospital Health Enterprise, Kristiansand, Norway. ${ }^{8}$ Department of Production Animal Clinical Sciences, Section of Small Ruminant Research, Norwegian University of Life Sciences, Sandnes, Norway. ${ }^{9}$ Department of Ecology, Grimsö Wildlife Research Station, Swedish University of Agricultural Sciences, Riddarhyttan, Sweden. ${ }^{10}$ Department of Applied Mathematics and Computer Science, Technical University of Denmark, Lyngby, Denmark. ${ }^{\bowtie}$ email: lenju@sund.ku.dk
} 
As ticks go through the different life stages, they feed on a variety of species such as birds, mammals and reptiles $^{12}$. In Scandinavia, the dominating vector for disease-causing pathogens in humans is the castor bean tick, Ixodes ricinus ${ }^{6,17}$. A generalist, this hard tick is a parasite of over 300 different host species, exposing it to reservoirs of multiple pathogens ${ }^{18-20}$. Immature life stages of $I$. ricinus feed on hosts of all sizes, whereas adult stages mainly feed on larger hosts, making ungulates and livestock important for maintaining these tick populations, also as a means for geographical dispersal ${ }^{12}$. Long distance dispersal of ticks and their associated pathogens by migrating passerine birds have been documented in several studies from Scandinavia ${ }^{21,22}$.

LB causing pathogens belong to the Borrelia burgdorferi sensu lato (s.l.) species complex, with the most common in southern Scandinavia being B. afzelii, B. garinii, B. burgdorferi sensu stricto (s.s.), B. spielmanii and B. valaisiana, with $B$. lusitaniae being relatively rare ${ }^{4,23-27}$. Species belonging to the $B$. burgdorferi s.l. complex are some of the main disease causing tick-borne pathogens in the northern hemisphere, and clinical manifestations differ between the different pathogen species ${ }^{28-30}$. Other disease-causing pathogens reported from the region are B. miyamotoi, Anaplasma phagocytophilum, Rickettsia helvetica, Neoehrlichia mikurensis, Babesia divergens, B. microti, B. venatorum, Bartonella henselae, and TBE-virus complex (TBEV $)^{4,31-37}$. A. phagocythopilum and the different Babesia species have long been known to cause disease in domestic livestock ${ }^{33}$, but may also cause disease in humans ${ }^{38-40}$. Although rarely, B. miyamotoi, R. helvetica and N. mikurensis have also been reported to cause disease in humans in southern Scandinavia ${ }^{41-44}$.

The risk of transmission of pathogens not only depends on the tick vector but also on reservoir hosts as some pathogens are associated with particular vertebrate species, whereas others are more generalists ${ }^{35}$. Thus, host species community composition may determine which pathogens are present and how prevalent they are. Within the B. burgdorferi s.l. species complex found in Scandinavia, most of the species appear to have rodents as their main reservoir hosts ${ }^{45}$. However, B. garinii and B. valaisiana have been found to be associated with birds ${ }^{19,45}$, and $B$. lusitaniae is more prevalent in certain lizard species (Lacerta agilis, Podarcis muralis) ${ }^{19,45}$. B. miyamotoi has mostly been associated with rodents ${ }^{18}$, whereas reservoir hosts for TBEV are still debated, but thought to be mainly rodents, small mammals and the tick vector themselves ${ }^{18,45,46}$. Rodents and wild ruminants have been found to be major reservoirs of A. phagocytophilum in the US and Asia ${ }^{33,47,48}$. Although found in rodents in Europe, wild ruminants seems to be the main reservoir ${ }^{33}$. Reservoir hosts for N. mikurensis have not yet been fully investigated, but is thought to be wild rodents ${ }^{49-51}$. The different Babesia species have different reservoirs, with $B$. divergens associating with bovines, $B$. microt $i$ with rodents and $B$. venatorum with cervids ${ }^{52}$. Although rodents and other mammals seem to be a reservoir for $R$. helvetica, the ticks themselves also serve as a major reservoir, with transovarial and sexual transmission ${ }^{18,53}$.

Prevalence of a given pathogen may not only be determined by the availability of suitable host species. Several studies have found a correlation between pathogen prevalence and tick abundance ${ }^{54-57}$, which in turn is affected by several factors, such as climate ${ }^{1,5}$, land cover, landscape composition, and availability and density of host species ${ }^{5,12}$. Climate and landscape may directly affect ticks and their life cycle ${ }^{1,5}$, but they may additionally affect ticks indirectly as these factors also influence their host species. I. ricinus, for example, have been found to have high abundance in forested habitats that provide optimal conditions for tick survival ${ }^{58-60}$. These forested habitats also provide optimal conditions for tick host species, particularly forest edge zones where the availability of both food and shelter can elevate local abundance of several host species ${ }^{60,61}$. Thus, the prevalence of specific pathogens in a region is a result of the complex interaction between ticks, their host species and the environment.

For vector-borne diseases it may be important to define zones with high pathogen prevalence as they pose a health-risk to both people and animals living within these areas ${ }^{62}$. Maps of disease prevalence can help pin-point high risk areas and finding geographical patterns within these maps may aid in determining causality or predicting potential outbreak areas ${ }^{63}$. The complex nature of tick-borne pathogen transmission cycles can complicate the development of predictive models of pathogen prevalence, as it can be difficult to obtain and include important factors driving these cycles. However, as environmental variables may affect both ticks, their hosts and habitat, environmental data can potentially be used directly and as proxies in predictive models ${ }^{64}$. Numerous studies have linked biotic and abiotic data to the prevalence of tick-borne pathogens ${ }^{64-66}$. Stefanoff et al. ${ }^{66}$ used temperature, precipitation, and various variables related to forest cover and unemployment rates in humans to predict high risk TBE areas in Poland, and Hönig et al. ${ }^{64}$ used environmental data to predict probability of tick infection for both LB and TBE in the Czech Republic and Germany. Randolph and Rodgers ${ }^{65}$ used pattern matching modelling to relate climatic variables to the distribution of TBEV in mainland Europe, Scandinavia and the Baltic States and then projected this model to future climate change scenarios.

In previous studies, we have successfully modelled the distribution and abundance of I. ricinus ticks in Scandinavia using predictive machine learning (ML) algorithms and environmental variables ${ }^{67,68}$. Applying the same methods to data on tick-borne pathogens, may enable us to create predictive models for pathogen prevalence for southern Scandinavia, contributing to our understanding of the variation we find in human disease incidence. The resulting risk maps from such models could help identify areas at risk for tick-borne disease transmission. Here we present data on prevalence for 15 different tick-borne pathogens in southern Scandinavia. The data stems from the first coordinated multi-country study in northern Europe to date, with 29,440 tick nymphs collected from 50 sites in Denmark, Norway and Sweden and are available from the figshare repository ${ }^{69}$. We also present predictive maps of pathogen prevalence within southern Scandinavia, developed using ML algorithms and environmental variables.

\section{Methods}

Study region, site selection and field study. The stratification of the study region, site selection and field study have all been described in previous studies ${ }^{67,68,70}$. For the stratification, we used Fourier processed satellite imagery of the maximum normalized difference vegetation index ${ }^{71}$ (NDVI) and land cover data from 
Corine $^{72}$ (all $1 \times 1 \mathrm{~km}$ resolution). Our study region was limited to southern Scandinavia including all of Denmark, southern Norway and south-eastern Sweden (see Figs. 1 and 2 in Kjær et al. ${ }^{70}$ ).

We randomly selected 30 sites (80\% forest and 20\% meadow) in Denmark, 30 sites in Norway and 30 sites in Sweden for tick collection. Each of these sites had alternative sites, in case ticks could not be collected at the original site. We furthermore added 20 random sites (with 10 alternatives) along the Oslo Fjord in Norway, as this was a region of interest. We collected tick nymphs at each site between 15. August to 30. September, 2016 and stored the collected nymphs on dry ice. In the laboratory, we stored all ticks at $-80{ }^{\circ} \mathrm{C}$ until use. For a more thorough description of site selection and collection method see Kjær et al. ${ }^{70}$.

\section{Laboratory methods}

DNA extraction and screening of tick-borne pathogens by real-time PCR. Methods for DNA extraction and pathogen screening have been thoroughly described elsewhere ${ }^{4,28,69}$, but a short description follows. We aggregated tick nymphs into pools of 10, washed and homogenized them, and then isolated genomic DNA using the Maxwell 16 LEV Blood DNA kit (Promega, Madison, Wisconsin, USA) on a Maxwell16 Instrument. We used the BioMark real-time PCR system (Fluidigm, San Francisco, California, USA) for high-throughput microfluidic real-time PCR. We screened for bacterial and parasitic tick-borne pathogens previously found in $I$. ricinus from Scandinavia, as well as some of the most common tick-borne pathogens found in Europe: $B$. miyamotoi, B. burgdorferi sensu lato, B. afzelii, B. burgdorferi sensu stricto, B. garinii, B. lusitaniae, B. spielmanii, B. valaisiana, A. phagocytophilum, N. mikurensis, The spotted fever group (SFG) rickettsiae, R. helvetica, Francisella tularensis, Coxiella burnetii, B. canis, B. divergens, B. microti, B. venatorum, and Bartonella henselae ${ }^{4}$. The identity of the tick species I. ricinus, I. persulcatus and Dermacentor reticulatus was confirmed by molecular methods. We analysed data using the Fluidigm real-time PCR analysis software to obtain crossing point (CP) values, and set cut-off values to $\mathrm{CP} \leq 28$. On each chip, we included one negative water control as well as Escherichia coli primers and probes for internal inhibition control ${ }^{4}$. The Fluidigm real-time PCR method has been validated through several studies ${ }^{4,22,28,73-75}$.

Statistical analysis. Pathogen prevalence. We calculated pool prevalence as the number of positive pools (10 nymphs per pool) out of the total number of pools for each pathogen at each site. To test for significant differences in pathogen pool prevalence between sites, we used Pearson's chi-squared test statistics (test of equal or given proportions). We furthermore ran a mixed model logistic regression on the 2,944 pools with site as a random effect, to test for differences between countries and between strata. We used R 3.5. $2^{76}$ for all statistical analyses.

The minimum infection rate (MIR) is often reported in pooled prevalence studies and is the number of positive pools divided by the total number of ticks tested. MIR is however, not only dependent on the true pathogen prevalence but also on the arbitrarily chosen pool size. With increasing pools sizes and pathogen prevalence, MIR will increasingly underestimate the true individual-level prevalence as positive pools will be increasingly likely to include more than one positive tick. Nevertheless, as pool size was constant for all sites (10 nymphs) in this study, MIR may simply be calculated by dividing pool prevalence with pool size.

Individual pathogen prevalence. We estimated the individual-level pathogen prevalence in tick nymphs at each site based on the number of positive pools and number of examined pools using method 3 from Cowing et al. ${ }^{77}$ that assumes $100 \%$ test sensitivity and specificity and fixed pool size. Exact confidence limits (CIs) were calculated based on binomial theory ${ }^{77}$.

Spatial analyses. To identify geographical clustering of pathogen prevalence we ran a global spatial autocorrelation test for each pathogen in ArcMap 10.6.1 ${ }^{78}$ (Global Moran's I). In our case, Global Moran's I measures spatial autocorrelation based on site location and the prevalence at the sites, and evaluates whether the global prevalence patterns observed are significantly clustered, dispersed or random. Prior to analysis the geographical coordinates of each site were transformed into a flat UTM projection (all coordinates were forced into UTM zone $32 \mathrm{~N}$ ).

Furthermore, we used the program SatScan ${ }^{79}$ and the rsatscan ${ }^{80}$ package in R 3.5.2 $2^{76}$ to identify potential local pathogen clusters. For each of the pathogens, we performed spatial scan statistics with a circular to elliptic scanning window, using the Bernoulli probability mode ${ }^{81}$ and a maximum spatial window size of less than or equal to $50 \%$ of the total population at risk. The analysis will look for significant geographical clusters where sites included in circular or ellipsoid areas on average have either higher (hotspots) or lower (coldspots) prevalence compared to the sites outside the ellipsoids. SatScan then calculates the relative risk as the estimated risk within the cluster divided by the estimated risk outside the cluster. To evaluate the clusters, we used the Gini coefficient ${ }^{82}$, which is a measure of heterogeneity of the collection of clusters. The Gini coefficient aids in deciding which clusters to report, by determining whether there is evidence for multiple smaller clusters or a large joint cluster. For this analysis we looked at the observed number of positive pools out of the total number of pools tested and not the estimated prevalence in individual nymphs. Again, we transformed our coordinates into a flat UTM projection (UTM zone $32 \mathrm{~N}$ ) before running SatScan.

Spatial modelling. We used R 3.5.2 $2^{76}$ (package caret $^{83}$ ) to run different ML algorithm models on our site-specific pathogen prevalence data. We ran boosted regression tree (BRT) models (both Gaussian and Poisson loss functions) as well as support vector regression (SVR), using variables from among 92 environmental predictors as well as additional predictor variables depicting fragmentation and amount of habitat edge for our forest and meadow sites. A thorough description of the predictor variables are found in Kjær et al. ${ }^{68}$. 
B. miyamotoi

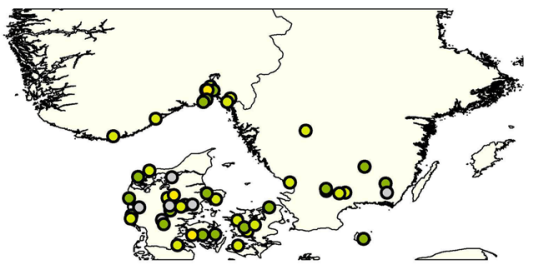

B. burgdorferi s.s.

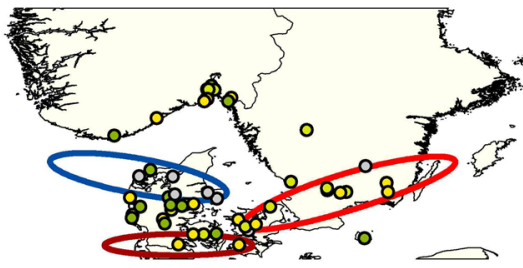

B. spielmanii

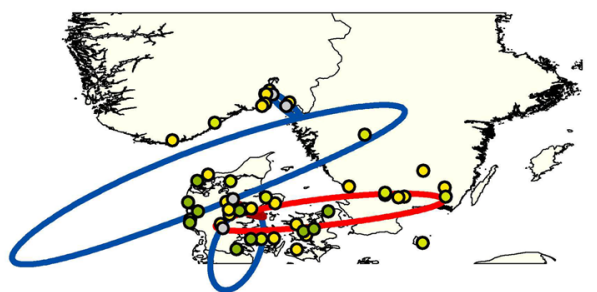

B. divergens

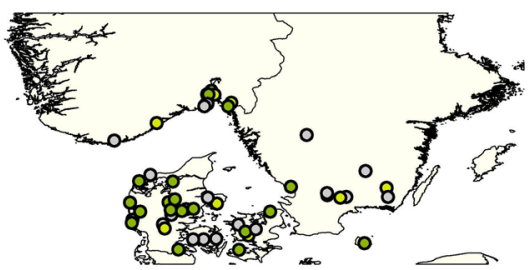

N. mikurensis

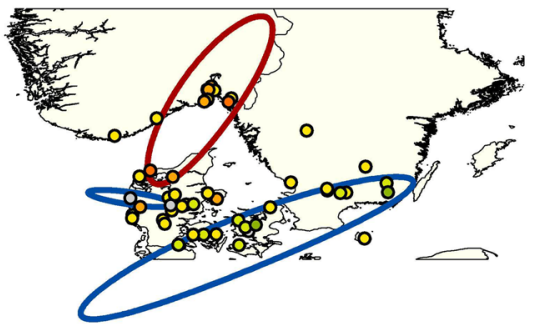

B. burgdorferi s.l.

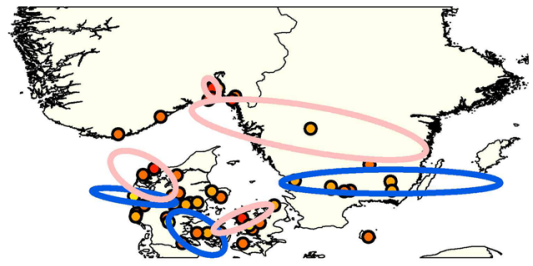

B. garinii

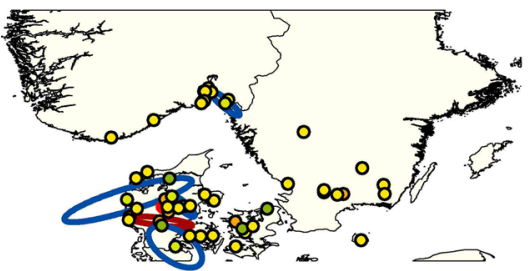

B. valaisiana

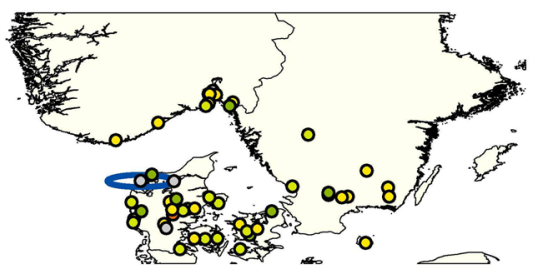

B. microti

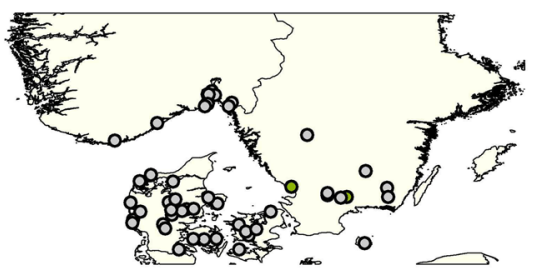

SFG rickettsiae

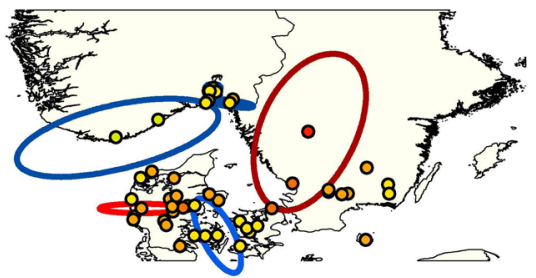

B. afzelii

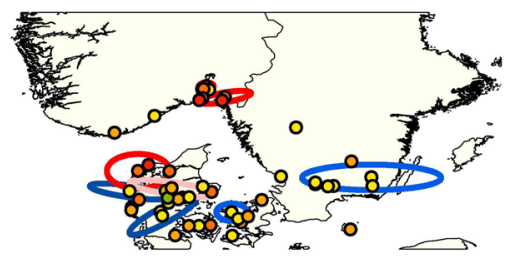

B. Iusitaniae

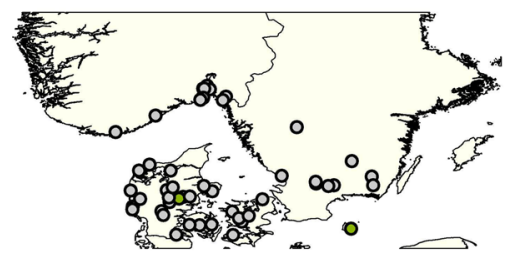

\section{A. phagocytophilum}

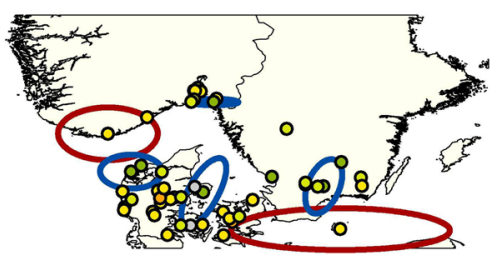

B. venatorum

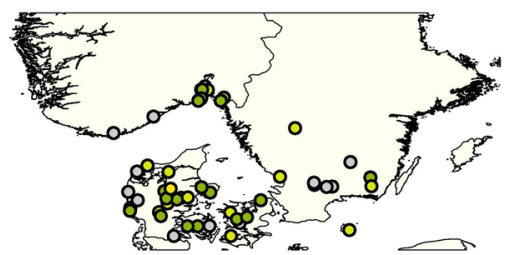

\section{R. helvetica}

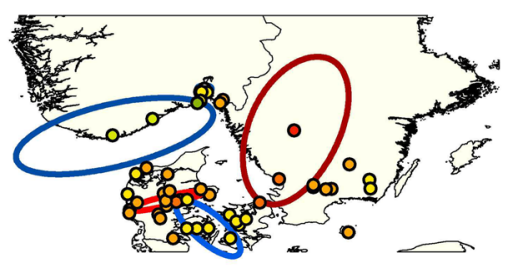

\section{Individual prevalence (\%)}
- absent
$\circ$
0.01-0.5 ०
1.0-5.0
- $10.0-20.0$
- 0-0.01
$0.5-1.0$
- 5.0-10.0
- 20.0-30.0

\section{Relative risk}

\section{$\longrightarrow$ 0-0.5 0.5-1.0 1.0-1.5 $-1.5-2.0 \longrightarrow 2.0$}

Figure 1. Individual prevalence. Individual prevalence at the 50 sample sites for the 15 pathogens found within the study region. Individual prevalence was calculated using method 3 from Cowling et al. ${ }^{77}$ that assumes $100 \%$ test sensitivity and specificity and fixed pool size. Clusters were analysed using SatScan on pool prevalence, and only significant clusters with the maximum Gini coefficient are depicted. Pool prevalence was calculated as positive pools out of the total number of pools at each site, whereas relative risk is calculated by SatScan as the estimated risk within a cluster divided by the estimated risk outside the cluster. 


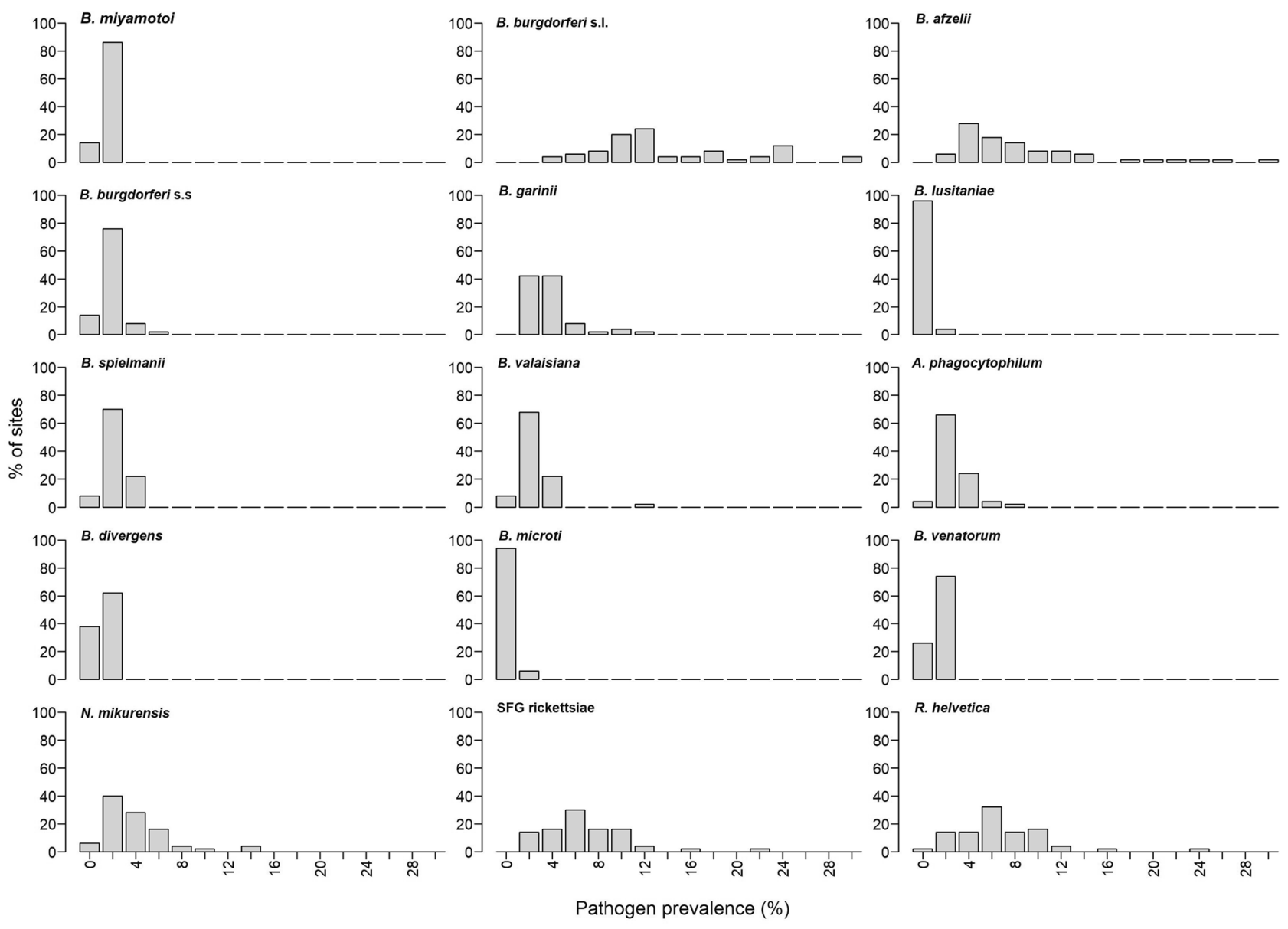

Figure 2. Pathogen prevalence ranges. Percentage of sites (50 total) having different ranges of individual pathogen prevalence for the 15 pathogens found within the study region.

BRT uses regression trees and gradient boosting ${ }^{84}$, whereas SVR is based on the support vector machine method that creates hyperplanes to separate data into classes. SVR tries to fit the error rate within a certain threshold from the hyper plane ${ }^{85}$. For the Gaussian BRT and the SVR, we transformed our pool prevalence data using the arcsine square root transformation. For the Poisson BRT we used the number of positive pools as the dependent variable, but added the total amount of pools as a weight. We ran a Pearson correlation test to remove predictor variables correlated more than $75 \%$. As SVRs are more sensitive than BRTs regarding the amount of variables compared to the amount of data points, we furthermore ran SVRs with recursive feature elimination ${ }^{86}$, leaving us with the most important model predictors for each pathogen.

For both the BRT and SVR models, we ran leave-one-out cross-validation (LOOCV) to validate our models. LOOCV is a method, where a model is fit while withholding one data point at a time, and the withheld "unknown" data points are then predicted by the model and thus used to evaluate model performance. LOOCV is often used when the sample size is too small to create a separate test set or to perform $k$-fold cross validation ${ }^{87}$. The LOOCV gives us an estimate of the prediction error in the form of the root-mean-square error, which we then normalized by dividing with the standard deviation of the observed data, resulting in a normalized rootmean-square error (NRMSE). For both the BRT and SVR models, we used a tuning grid to optimize model parameters, but held the number of trees for the BRT models constant at 1500 trees. If any of the final models proved to have suitable predictive power, they were used to predict and map prevalence to the entire study region. To draw the predictive maps, we used ArcMap 10.6.1 ${ }^{78}$.

\section{Results}

Field study. Due to logistic reasons and time restraints, we collected 29,440 tick nymphs from a total of 50 sites with 30 sites in Denmark, 11 sites in Norway and 9 sites in Sweden (Fig. 2 in Kjær et al. ${ }^{70}$ ). Coordinates of these sites and number of ticks collected are available from the figshare repository ${ }^{69}$, and the dataset has been described in Kjær et al. ${ }^{70}$.

Pathogen prevalence. In this study, a PCR run was deemed valid when all E. coli controls were positive, all water controls were negative, all amplification curves were accepted by the Fluidigm software algorithm for 


\begin{tabular}{|l|c|l|l|}
\hline Pathogen & \multicolumn{1}{|c|}{$\chi^{\mathbf{2}}$} & Df & P value \\
\hline B. miyamotoi & 83.27 & 49 & $<0.01$ \\
\hline B. afzelii & 655.92 & 49 & $<0.0001$ \\
\hline B. burgdorferi s.s & 241.57 & 49 & $<0.0001$ \\
\hline B. garinii & 399.29 & 49 & $<0.0001$ \\
\hline B. lusitaniae & 78.86 & 49 & $<0.01$ \\
\hline B. spielmanii & 209.84 & 49 & $<0.0001$ \\
\hline B. valaisiana & 364.36 & 49 & $<0.0001$ \\
\hline A. phagocytophilum & 348.04 & 49 & $<0.0001$ \\
\hline B. divergens & 61.04 & 49 & 0.12 \\
\hline B. microti & 46.67 & 49 & 0.57 \\
\hline B. venatorum & 65.69 & 49 & 0.056 \\
\hline N. mikurensis & 555.36 & 49 & $<0.0001$ \\
\hline R. helvetica & 483.17 & 49 & $<0.0001$ \\
\hline
\end{tabular}

Table 1. Pearson's chi square test for equality of proportions without continuity correction, testing for significant differences in pathogen prevalence (pool prevalence) between the 50 sites in Denmark, Norway and Sweden. Only tick-borne pathogens identified from I. ricinus ticks collected from Denmark, Norway and Sweden, 2016 are depicted (excluding B. burgdorferi s.l. and SFG rickettsiae).

ideal curves, and CP values were less than or equal to 28. All 2,944 pools tested positive for I. ricinus only ${ }^{17}$. F. tularensis, C. burnetii, B. canis, or B. henselae were not detected in any of the 2,944 pools.

Data on presence/absence of pathogens for each pool within a site are available from the figshare repository ${ }^{69}$. Each of the 50 sites tested positive for at least one of the 15 pathogens identified in the survey (including $B$. burgdorferi s.l. and SFG rickettsiae) at varying prevalence. Each site had an overall pool prevalence of at least $33 \%$ (pools positive for one or more pathogens). The pathogens found were (in order of highest to lowest overall mean prevalence) B. burgdorferi s.l. (mean pool prevalence $=69.9 \%$, range: $30-96.7 \%$ ), B. afzelii (mean pool prevalence $=48.2 \%$, range: $3.3-96.7 \%)$, $R$. helvetica $($ mean pool prevalence $=41.1 \%$, range: $0.0-91.7 \%)$, SFG rickettsiae (mean pool prevalence $=41.6 \%$, range: $1.7-90.0 \%$ ), B. garinii (mean pool prevalence $=23.5 \%$, range: 1.7-68.3), N. mikurensis (mean pool prevalence $=23.3 \%$, range: $0.0-75.0 \%)$, A. phagocytophilum (mean pool prevalence $=14.1 \%$, range: $0.0-50.0 \%), B$. valaisiana $($ mean pool prevalence $=12.2 \%$, range: $0.0-70.0 \%), B$. spielmanii (mean pool prevalence $=11.3 \%$, range: $0.0-30.0 \%)$, B. burgdorferi s.s. (mean pool prevalence $=8.5 \%$, range: $0.0-40.0 \%)$, B. miyamotoi (mean pool prevalence $=4.0 \%$, range: $0.0-16.7 \%)$, B. venatorum $($ mean pool prevalence $=3.2 \%$, range: $0.0-10.0 \%), B$. divergens (mean pool prevalence $=1.8 \%$, range: $0.0-6.9 \%)$, B. microti (mean pool prevalence $=0.1 \%$, range: $0.0-1.7 \%$ ), and $B$. lusitaniae (mean pool prevalence $=0.1 \%$, range: $0.0-3.3 \%$ ).

The Pearson's chi square test, to test for equality of proportions among the sites, showed significant differences in prevalence between sites for all pathogens except the three relatively rare Babesia species-B. divergens, $B$. venatorum, and B. microti (Table 1 ).

The mixed model logistic regression showed that only pool prevalence of $B$. miyamotoi had an effect of stratum when taking site variation into account. Here, prevalence differed significantly between all strata $(P<0.001)$ with higher values in strata with low NDVI. Meadow sites with low NDVI had the highest pool prevalence. We also tested for differences between countries and found significantly higher prevalence in Norway compared to Denmark and Sweden for B. burgdorferi s.l., B. afzelii, and N. mikurensis (all $P<0.001$ ). This pattern was reversed for $R$. helvetica and SFG rickettsiae, with prevalence being significantly lower in Norway than in Denmark and Sweden $(P<0.001)$.

Individual prevalence. An overview of the estimated specific pathogen prevalence in individual nymphs and frequencies are found in Figs. 1 and 2. All estimated site-level individual prevalence and CI's are available from the figshare repository ${ }^{69}$. Table 2 describes the overall mean and range of all the pathogens found in this study. Overall, the B. burgdorferi sensu lato complex had the highest mean prevalence (13.0\%), and was found at all sites. B. afzelii contributed mostly to this pattern with a mean prevalence of $7.9 \%$ (all sites testing positive), followed by B. garinii with $2.9 \%$ (all sites testing positive), B. valaisiana with $1.4 \%$ (positive at $92 \%$ of the sites), B. spielmanii with $1.2 \%$ (positive at $92 \%$ of the sites), and B. burgdorferi s.s. with a mean prevalence of $0.9 \%$ (positive at $86 \%$ of the sites). B. lusitaniae was only found at two sites, both in Denmark, in both cases with low prevalence $(0.2 \%$ and $0.3 \%)$. B. miyamotoi had a mean prevalence of $0.4 \%$ (positive at $86 \%$ of the sites), and mean prevalence for A. phagocytophilum was $1.6 \%$ (positive at $96 \%$ of the sites). Mean prevalence was $0.2 \%$ for $B$. divergens (positive at $62 \%$ of the sites), and $0.3 \%$ for B. venatorum (positive at $74 \%$ of the sites). B. microti was found with low prevalence at three sites in the Skåne region in Sweden (all 0.2\%). N. mikurensis had a mean prevalence of $3.0 \%$ (positive at $94 \%$ of the sites), with $5.8 \%$ for SFG rickettsiae (positive at $100 \%$ of the sites), and $5.8 \%$ for R. helvetica (positive at $98 \%$ of the sites).

Spatial analysis. Results from the global Moran's I only identified B. afzelii, B. microti, N. mikurensis and $R$. helvetica as having a clustered prevalence patterns where neighbouring sites tended to have more similar 


\begin{tabular}{|l|l|l|}
\hline Pathogen & Average (\%) & Range (\%) \\
\hline B miyamotoi & 0.4 & $0.0-1.8$ \\
\hline B. burgdorferi s.l & 13.0 & $3.5-28.8$ \\
\hline B. afzelii & 7.9 & $0.3-28.8$ \\
\hline B. burgdorferi s.s & 0.9 & $0.0-5.0$ \\
\hline B. garinii & 2.9 & $0.2-9.1$ \\
\hline B. lusitaniae & 0.01 & $0.0-0.3$ \\
\hline B. spielmanii & 1.2 & $0.0-3.5$ \\
\hline B. valaisiana & 1.4 & $0.0-11.3$ \\
\hline A. phagocytophilum & 1.6 & $0.0-6.7$ \\
\hline B. divergens & 0.2 & $0.0-0.7$ \\
\hline B. microti & 0.01 & $0.0-0.2$ \\
\hline B. venatorum & 0.3 & $0.0-1.1$ \\
\hline N. mikurensis & 3.0 & $0.0-12.9$ \\
\hline SFG rickettsiae & 5.8 & $0.2-20.6$ \\
\hline R. helvetica & 5.8 & $0.0-22.0$ \\
\hline
\end{tabular}

Table 2. Average and range of pathogen prevalence (estimated individual nymph prevalence) in southern Scandinavia. Only the 15 tick-borne pathogens identified from I. ricinus ticks collected from Denmark, Norway and Sweden, 2016 are depicted (including B. burgdorferi s.l. and SFG rickettsiae).

\begin{tabular}{|l|l|l|l|}
\hline Pathogen & Moran's index & z-score & $P$ value \\
\hline B. miyamotoi & -0.01 & 0.08 & 0.93 \\
\hline B. afzelii & 0.34 & 4.85 & $<0.0001$ \\
\hline B. burgdorferi s.s & 0.10 & 0.62 & 0.10 \\
\hline B. garinii & -0.03 & -0.07 & 0.94 \\
\hline B. lusitaniae & -0.02 & 0.03 & 0.98 \\
\hline B. spielmanii & -0.08 & -0.79 & 0.43 \\
\hline B. valaisiana & -0.08 & 49 & 0.33 \\
\hline A. phagocytophilum & 0.01 & 0.43 & 0.67 \\
\hline B. divergens & -0.07 & -0.63 & 0.53 \\
\hline B. microti & 0.14 & 2.45 & 0.014 \\
\hline B. venatorum & -0.03 & -0.14 & 0.89 \\
\hline N. mikurensis & 0.36 & 5.11 & $<0.0001$ \\
\hline R. helvetica & 0.47 & 6.68 & $<0.0001$ \\
\hline
\end{tabular}

Table 3. Global Moran's I test, testing for spatial autocorrelation of pathogen prevalence (pool prevalence) between the 50 sites in Denmark, Norway and Sweden. Only tick-borne pathogens identified from I. ricinus ticks collected from Denmark, Norway and Sweden, 2016 are depicted (excluding B. burgdorferi s.l. and SFG rickettsiae).

prevalence than expected from a random distribution $(P<0.05$, Table 3$)$. The global prevalence patterns of the remaining pathogens did not appear to differ significantly from a random geographical pattern (Table 3 ).

The SatScan local spatial analysis detected significant clusters within the region for the B. burgdorferi s.l. complex, hereunder for B. afzelii, B. burgdorferi s.s., B. garnii, B. spielmanii, and B. valaisiana (Fig. 1, Supplementary Table S1 online). The analysis also detected significant clusters for A. phagocytophilum, N. mikurensis, and SFG rickettsiae and $R$. helvetica. Prevalence hot spots and cold spots were interspersed for most of the pathogens, with no obvious interpretable overall geographical patterns. For B. burgdorferi s.s., B. spielmanii, N. mikurensis and $R$. helvetica some clusters even connected sites, separated by large water bodies, further indicating that no clear patterns were discernible. The number of sites within hot spots ranged from 1-11, and ranged from 1-15 within cold spots (Supplementary Table S2 online).

Spatial modelling. We created BRT and SVR models for 13 of the 15 pathogens found in the region (including B. burgdorferi s.l. and SFG rickettsiae). B. lusitaniae and B. microti were both highly zero-inflated as only two and three sites were positive for these pathogens respectively, and were thus omitted from the analyses. Removing highly correlated variables (>75\%) resulted in 56 predictors used in both the BRT and SVR models. NRMSE, and $\mathrm{R}^{2}$-values for all final best models can be found in Table 4. Except for R. helvetica and N. mikurensis, the pathogen models generally performed poorly with NRMSE-values $>0.85$ and $\mathrm{R}^{2}$-values ranging from 


\begin{tabular}{|l|l|l|l|l|l|l|l|}
\hline Pathogen & SVM model type & $\mathbf{R}^{2}$ & NRMSE & \# variables & BRT model & $\mathbf{R}^{2}$ & NRMSE \\
\hline B. miyamotoi & Polynomial kernel & 0.07 & 1.01 & $65^{\text {a }}$ & Poisson & 0.06 & 1.17 \\
\hline B. burgdorferi s.l & Linear kernel & 0.22 & 0.89 & 65 & Gaussian & 0.05 & 1.05 \\
\hline B. afzelii & Polynomial kernel & 0.24 & 0.89 & 40 & Gaussian & 0.23 & 0.89 \\
\hline B. burgdorferi s.s & Linear kernel & 0.07 & 0.96 & $65^{\text {a }}$ & Gaussian & 0.11 & 0.96 \\
\hline B. garinii & Polynomial kernel & 0.03 & 0.99 & 50 & Gaussian & 0.05 & 1.25 \\
\hline B. spielmanii & Radial kernel & 0.00 & 0.97 & $65^{\text {a }}$ & Gaussian & 0.15 & 1.29 \\
\hline B. valaisiana & Radial kernel & 0.21 & 1.03 & $65^{\text {a }}$ & Gaussian & 0.01 & 1.10 \\
\hline A. phagocythophilum & Radial kernel & 0.19 & 0.90 & 5 & Gaussian & 0.04 & 1.04 \\
\hline B. divergens & Polynomial kernel & 0.08 & 0.95 & 20 & Gaussian & 0.02 & 1.19 \\
\hline B. venatorum & Polynomial kernel & 0.17 & 0.93 & 30 & Gaussian & 0.17 & 0.92 \\
\hline N. mikurensis & Polynomial kernel & 0.43 & 0.75 & 10 & Gaussian & 0.29 & 0.84 \\
\hline SFG rickettsiae & Linear kernel & 0.45 & 0.72 & 5 & Gaussian & 0.40 & 0.77 \\
\hline R. helvetia & Linear kernel & 0.48 & 0.74 & 10 & Gaussian & 0.41 & 0.76 \\
\hline
\end{tabular}

Table 4. Comparison of the best SVR and BRT models (lowest NRMSE) for each of the 13 pathogens (excluding B. microti and B. lusitaniae). NRMSE is the normalized root-mean-square error (root-mean-square

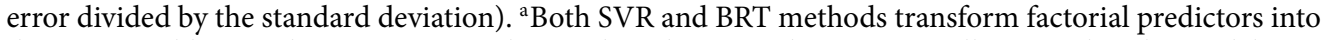
dummy variables, resulting in more predictors than the 56 predictors originally entered in the models.

$0.002-0.24$, and were consequently not used to create prediction maps for the region. In general, the SVR models performed better than the BRT models and the BRT models with Gaussian loss performed better that the models with Poisson loss. Tuning grid values for all final best models can be found in the Supplementary Table S2 online.

The best and final models for N. mikurensis and R. helvetica were the SVR models with polynomial and linear kernels respectively. Both of these models ended up with a total of ten predictors, selected via the recursive feature elimination method (See Supplementary Figs. S1 and S2 online). The models resulted in NRMSE values of 0.75 and 0.74 and $\mathrm{R}^{2}$ values of 0.43 and 0.48 , respectively (Fig. 3 ). We plotted the prediction errors for both models (observed pool prevalence - predicted pool prevalence, both arcsine-square-root transformed) for the LOOCV predictions, to check for potential spatial patterns, and did not observe any clear patterns (See Supplementary Fig. S3 online). The final prediction maps included all of Denmark, $68.4 \%$ of Norway and $85.8 \%$ of Sweden (Fig. 4). The maps show relatively high prevalence of $N$. mikurensis in the north-eastern parts of Sweden as well as the eastern and north-eastern coastline of Norway. R. helvetica prevalence was high throughout Denmark and Sweden, but low in Norway, except the northern parts of the Oslo Fjord.

The ten predictors driving both models were daytime temperature and related parameters, the vegetation indexes NDVI and EVI and their related parameters, and parameters related to the middle infra-red index (See Supplementary Figs. S1 and S2 online). In the model for N. mikurensis, land cover ranked in 4th place, but mostly due to moors and heathland (See Supplementary Fig. S1 online).

\section{Discussion}

We tested 29,440 I. ricinus nymphs for 19 different pathogens in 2,944 pools (including B. burgdorferi s.l. and SFG rickettsiae). We found a high pathogen infection in the collected ticks, corresponding to what has been found for the region in other studies ${ }^{4,15,23,25,28,54,88-90}$. Previous studies have found overall prevalence of the $B$. burgdorferi s.l. complex ranging from 10-15\% in tick nymphs from recorded studies in Denmark, Norway and Sweden $^{15,26,89-92}$, with local prevalence as high as $33.1 \%$ in southern Norway ${ }^{27}$. In our study, estimated individual prevalence over $20 \%$ were found along the Oslo Fjord in Norway, and at two sites in Jutland and one on Zealand in Denmark. B. lusitaniae was only found at two sites in Denmark, Jutland and Bornholm, but has previously been reported from northern Zealand in Denmark ${ }^{26}$, and Östergötland in Sweden ${ }^{90}$. Our estimated prevalence for $A$. phagocytophilum was also within the recorded prevalence range of $1-24 \%$ in tick nymphs for the region ${ }^{15,23,33,89,93}$. The overall prevalence for the Babesia spp. were $\leq 1 \%$ whereas other studies from the region have reported prevalence of Babesia spp. up to 3.6\%,28,94,95. Reported prevalence in tick nymphs for N. mikurensis have ranged from $0-19 \%$ for the region ${ }^{4,15,28,31,50,96,97}$, with high prevalence in southern Norway, particularly along the Oslo Fjord $^{15}$. Our estimated prevalence was well within the reported range, also with high local prevalence along the Oslo Fjord in Norway. All estimated prevalence for N. mikurensis higher than 5\% was found along the Oslo Fjord and in Jutland in Denmark. A study from 2015 recorded $R$. helvetica for the first time in Norway in one sample of adult ticks ${ }^{89}$, and has since been found to be widespread at a low prevalence in southern Norway (Kjelland, unpublished data). R. helvetica has previously been recorded in tick nymphs from Denmark and Sweden with prevalence ranging from to $1.4-18 \%{ }^{93,98}$. Our prevalence was within that range except for a prevalence of $22 \%$ at one site in Sweden. Interestingly, we found $R$. helvetica at all sites except one site in Norway, supporting that although $R$. helvetica was detected only recently in Norway, the pathogen is common in southern Norway at prevalence $\leq 5 \%$. The significantly lower pool prevalence of $R$. helvetica in Norway compared to Denmark and Sweden, and the SatScan analysis showing a cold spot for $R$. helvetica in Norway, could also suggest that the presence of $R$. helvetica in Norway may be of newer origin. We did not find F. tularensis, B. canis or B. henselae at any of our sites, however our survey does not rule out the presence of these pathogens in the study area albeit 

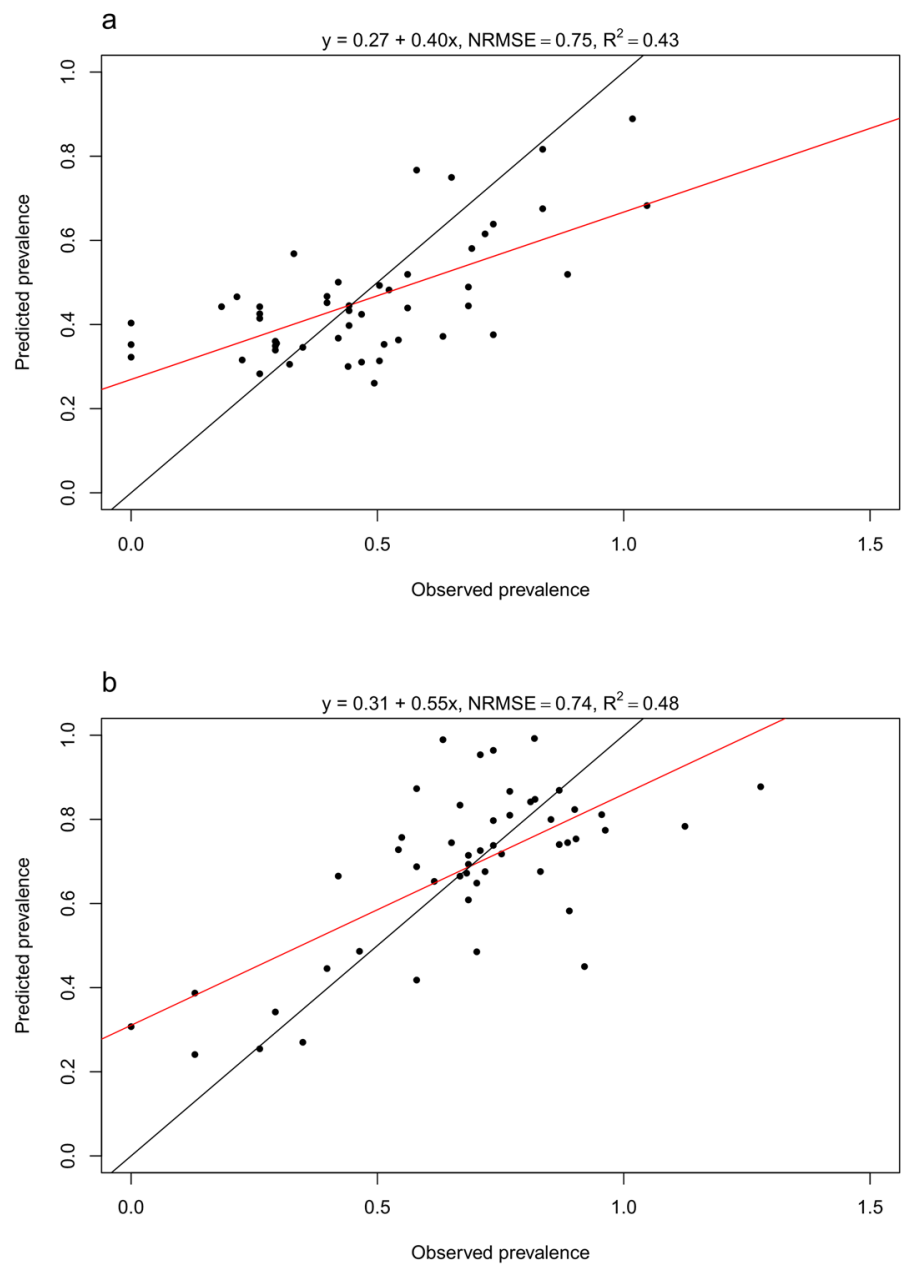

Figure 3. Observed versus predicted prevalence. Observed prevalence plotted against predicted prevalence (pool prevalence, both arc-sine-square-root transformed) for (a) N. mikurensis and (b) R. helvetica, based on LOOCV results from the final best SVR models. The red line is a linear regression line on the observed and predicted values, and the black line depicts a 1:1 relationship between observed and predicted values. NRMSE is the normalized root-mean-square error.

at low prevalence ${ }^{17}$. B. canis is transmitted via the meadow tick (D. reticulatus) $)^{99}$, and as all our collected ticks were I. ricinus, we did not expect to find this pathogen.

Except for the three Babesia species, we found a significant difference in pathogen prevalence between sites. These differences indicate that environmental factors may be driving the different prevalence at the specific sites. We sampled all sites within the same period using the same field protocol, thus any difference in prevalence should be a result of some form of causality. The pathogens with no differences between sites generally also had very low prevalence and thus low statistical power to detect potential differences between sites. This especially applies to B. microti which is considered absent from most of the study area except southern Sweden ${ }^{94}$ where we also found it in this study. The interplay between ticks, host species and the environment is complex, but finding drivers of pathogen prevalence may aid in creating future predictive models and risk maps for unsampled regions. However, it can be challenging to determine drivers of the different pathogens, causing the observed patterns. Some of our sites may have differed in the abundance of rodent species, causing differences in the prevalence of several pathogen species having rodents as reservoirs ${ }^{45,52}$. Sites with relatively high abundance of different bird species could explain prevalence patterns of B. garinii and B. valaisiana ${ }^{19,45}$, and the presence and abundance of cervids at our sites could impact the prevalence of $B$. venatorum ${ }^{45}$. R. helvetica, A. phagocytophilum and possibly $N$. mikurensis may have a broader spectrum of host species ${ }^{18,31,47,53}$, which could explain why we found these pathogens throughout the region.

To further look for causal effects, we conducted two geographical cluster analyses. The Global Moran's I showed significant clustering for B. afzelii, B. microti (only two sites were positive for this pathogen), N. mikurensis and $R$. helvetica, suggesting that nearby sites had similar suitability for these pathogens. $B$. microti has recently been reported from the central part of southern Sweden but was never reported in Denmark and Norway, suggesting this area may be a hot spot for B. microti in Scandinavia. We furthermore investigated whether we could identify clusters of neighbouring sites where prevalence was significantly higher or lower compared to surrounding areas. We were expecting some form of overall latitudinal or environmental gradient that correlated 


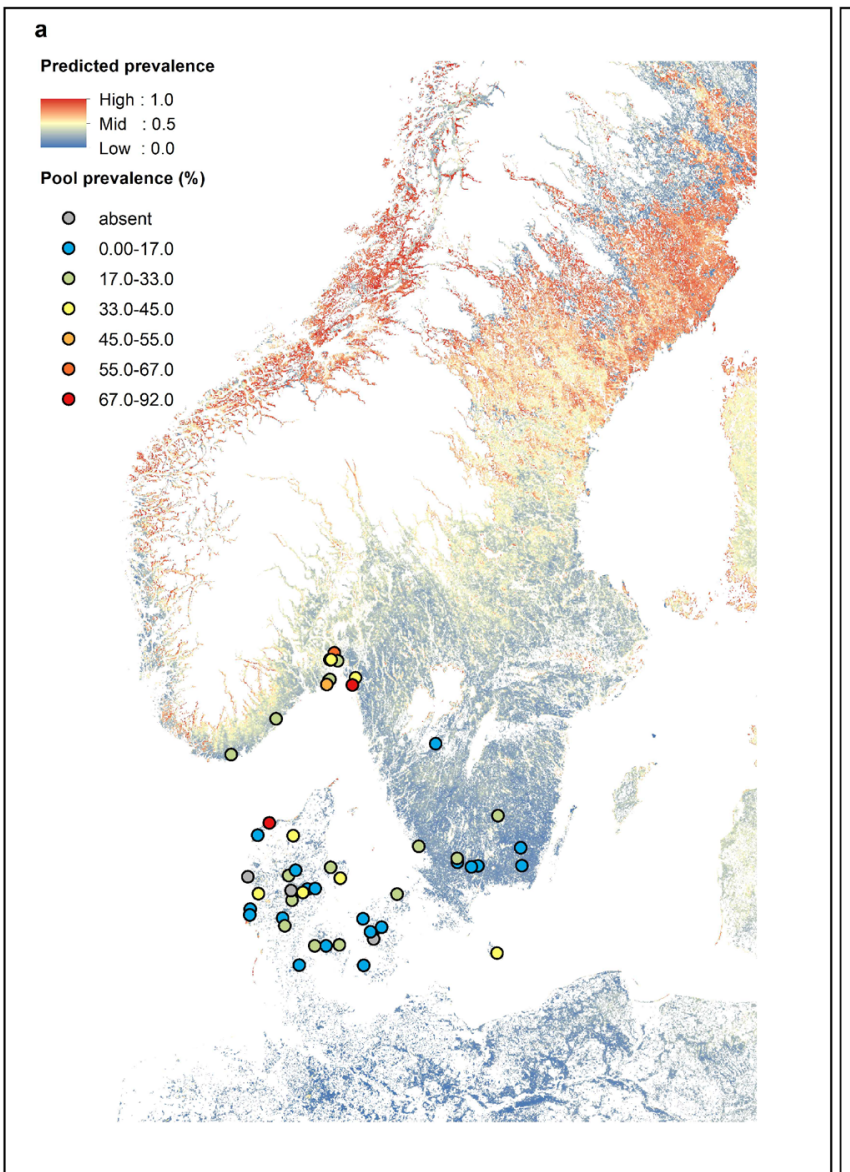

b

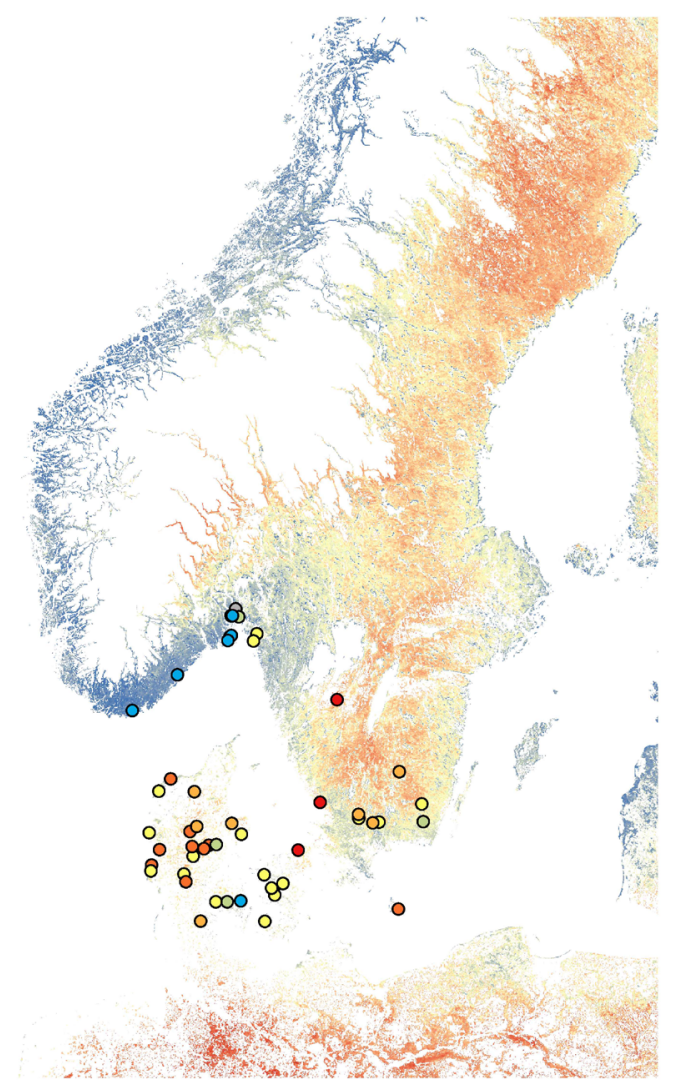

Figure 4. Prevalence maps. Maps of predicted prevalence (pool prevalence, back-transformed from arc-sinesquare-root) for (a) N. mikurensis and (b) R. helvetica from the final SVR models. Observed pool prevalence at the 50 study sites is also depicted. White areas are altitudes above $450 \mathrm{~m}$ or lakes, rivers and streams, or habitats other than forest or meadow (not predicted). The maps were created using ArcMap 10.6.1 ${ }^{78}$.

with the distribution of key environmental or climatic variables. The geographical clusters we found however, did not show a clear interpretable pattern, with hotspots and cold spots of prevalence dispersed throughout the region. Previous studies have found significant effects of environmental variables (temperature, NDVI, land cover) on the spatial variation in the occurrence of TBEV ${ }^{65,100,101}$. Transmission of TBEV is thought to be dependent on co-feeding of larvae and nymphs on hosts, and thus the synchrony of the two instars ${ }^{100,102,103}$. This synchrony is determined by the tick life cycle which in turn is affected by environmental conditions ${ }^{12,100}$. The transmission of bacteria and parasites, however, may be more dependent on the availability of competent host species and factors affecting this availability ${ }^{47,100,104}$. While our two spatial cluster analyses proved that there is significant geographical clustering for some of the pathogens analysed, we were not able to discern any obvious single predictors associated geographically with these clusters.

The lack of discernible predictors could explain why our predictive ML pathogen models had poor predictive power. As tick abundance is thought to affect pathogen prevalence ${ }^{54,55,57}$, we tried to run our ML models with observed abundance of different tick instars at each site ${ }^{68}$ (data not presented), however, this did not improve the models, and these abundance predictors were thus left out of the final models. Only the models for $N$. mikurensis and $R$. helvetica had moderate predictive power and were the only models used to create predictive maps. Abundance and/or diversity of host species for these two pathogens may be affected by environmental variables that could act as proxies in the models. The models for both $N$. mikurensis and $R$. helvetica were driven by temperature and vegetation parameters and when comparing predicted prevalence to the actual prevalence data (Fig. 4), the models predicted well for known areas in Denmark and the southern parts of Norway and Sweden. We did not test ticks from northern Sweden and north-eastern Norway, thus predictions for these areas should be interpreted with caution. However, Jenkins et al. ${ }^{50}$ found N. mikurensis prevalence of ca. $6.5 \%$ in the north-western parts of Norway, thus our models may still be useful for these areas. Both prediction maps show high predicted prevalence of both $N$. mikurensis and R. helvetica in northern Sweden above the biogeographical and climatic boundary called Limes Norrlandicus $(\mathrm{LN})^{105}$. In our previous predictive modelling studies ${ }^{67,68}$, we found that LN reflected boundaries for tick distribution and abundance, and thus predictions of high pathogen prevalence in areas above this boundary are questionable. Low geographical spread of our data in general could explain the low predictive power of most of our pathogen models. Low sample size could also explain the low 
model performances, as a low number of data points with potential low variability in the predictors may not be sufficient for the ML methods to learn patterns and dependencies between the data and the predictors. The use of LOOCV may additionally lead to overfitting and thus low generalisability to unseen data, reducing the predictive power. Poor model performance could also be due to absence of key pathogen drivers such as data on host species composition and abundance, which our environmental data could not act as proxies for. As data on host species can be hard to obtain at a high resolution, it may complicate predictive modelling of certain pathogens. We do not know how stable the observed prevalence is and if the pattern we found in 2016, would also be observed in later years. If the differences we observed in 2016 are not due to differences in host species composition (which can also fluctuate between years) or other potential drivers, but instead are due to temporal fluctuations and epidemics in the wild hosts, it will complicate predictive modelling and mapping.

\section{Conclusion}

We found high prevalence of tick-borne pathogens in tick nymphs from southern Scandinavia. Particularly Borrelia spp., N. mikurensis, and R. helvetica were widespread throughout the region. Although R. helvetica has only recently been found in Norway, we found that the pathogen was common throughout Norway, albeit at prevalence $\leq 5 \%$. Significant differences in prevalence between sites and geographically interspersed clusters with high and low prevalence, suggest highly complex patterns, which complicates creating predictive models of pathogen prevalence.

\section{Data availability}

All data generated or analysed during this study are available on figshare (https://doi.org/10.6084/m9.figsh are.c. $4938270 . v 1)$.

Received: 20 July 2020; Accepted: 27 October 2020

Published online: 09 November 2020

\section{References}

1. Pfäffle, M., Littwin, N., Muders, S. V. \& Petney, T. N. The ecology of tick-borne diseases. Int. J. Parasitol. 43, 1059-1077 (2013).

2. Han, B. A. \& Yang, L. Predicting novel tick vectors of zoonotic disease. in ICML Workshop on \#Data4Good: Machine Learning in Social Good Applications 71-75 (2016).

3. de la Fuente, J., Estrada-Pena, A., Venzal, J. M., Kocan, K. M. \& Sonenshine, D. E. Overview: ticks as vectors of pathogens that cause disease in humans and animals. Front. Biosci. 13, 6938-6946 (2008).

4. Michelet, L. et al. High-throughput screening of tick-borne pathogens in Europe. Front. Cell. Infect. Microbiol. 4, 103 (2014).

5. Estrada-Peña, A. \& de la Fuente, J. The ecology of ticks and epidemiology of tick-borne viral diseases. Antiviral Res. 108, 104-128 (2014).

6. Paul, R. E. L. et al. Environmental factors influencing tick densities over seven years in a French suburban forest. Parasit. Vectors 9, 309 (2016).

7. Randolph, S. E. Tick-borne disease systems emerge from the shadows: the beauty lies in molecular detail, the message in epidemiology. Parasitology 136, 1403 (2009).

8. Jore, S. et al. Multi-source analysis reveals latitudinal and altitudinal shifts in range of Ixodes ricinus at its northern distribution limit. Parasit. Vectors 4, 1-11 (2011).

9. Bernstein, L. et al. Climate Change 2007: Synthesis Report. Contribution of working groups I, II and III to the fourth assessment report of the Intergovernmental Panel on Climate Change. Intergovernmental Panel on Climate Change [Core Writing Team, Pachauri, R.K and Reisinger, A. (eds.)] https://www.ipcc.ch/pdf/assessment-report/ar4/syr/ar4_syr_full_report.pdf (2007).

10. Kovats, R. S., Campbell-Lendrum, D. H., McMichael, A. J., Woodward, A. \& Cox, J. S. Early effects of climate change: do they include changes in vector-borne disease?. Philos. Trans. R. Soc. Lond. B. Biol. Sci. 356, 1057-1068 (2001).

11. Gage, K. L., Burkot, T. R., Eisen, R. J. \& Hayes, E. B. Climate and vectorborne diseases. Am. J. Prev. Med. 35, 436-450 (2008).

12. Medlock, J. M. et al. Driving forces for changes in geographical distribution of Ixodes ricinus ticks in Europe. Parasit. Vectors 6, 1-11 (2013).

13. Andreassen, A. et al. Prevalence of tick borne encephalitis virus in tick nymphs in relation to climatic factors on the southern coast of Norway. Parasit. Vectors 5, 1-12 (2012).

14. Soleng, A. et al. Distribution of Ixodes ricinus ticks and prevalence of tick-borne encephalitis virus among questing ticks in the Arctic Circle region of northern Norway. Ticks Tick. Borne. Dis. 9, 97-103 (2018).

15. Kjelland, V. et al. Tick-borne encephalitis virus, Borrelia burgdorferi sensu lato, Borrelia miyamotoi, Anaplasma phagocytophilum and Candidatus Neoehrlichia mikurensis in Ixodes ricinus ticks collected from recreational islands in southern Norway. Ticks Tick. Borne. Dis. 9, 1098-1102 (2018).

16. Paulsen, K. M. et al. Prevalence of tick-borne encephalitis virus in Ixodes ricinus ticks from three islands in north-western Norway. APMIS 123, 759-764 (2015).

17. Kjær, L. J. et al. A large-scale screening for the taiga tick, Ixodes persulcatus, and the meadow tick, Dermacentor reticulatus, in southern Scandinavia, 2016. Parasit. Vectors 12, 338 (2019).

18. Oechslin, C. P. et al. Prevalence of tick-borne pathogens in questing Ixodes ricinus ticks in urban and suburban areas of Switzerland. Parasit. Vectors 10, 558 (2017).

19. Becker, N. S. et al. Recurrent evolution of host and vector association in bacteria of the Borrelia burgdorferi sensu lato species complex. BMC Genom. 17, 734 (2016).

20. Bowman, A. S. \& Nuttall, P. A. Ticks: Biology, Disease and Control (Cambridge University Press, Cambridge, 2004).

21. Hasle, G. et al. Transport of ticks by migratory passerine birds to Norway. J. Parasitol. 95, 1342-1351 (2009).

22. Klitgaard, K. et al. Screening for multiple tick-borne pathogens in Ixodes ricinus ticks from birds in Denmark during spring and autumn migration seasons. Ticks Tick. Borne. Dis. 10, 546-552 (2019).

23. Skarphédinsson, S. et al. Detection and identification of Anaplasma phagocytophilum, Borrelia burgdorferi, and Rickettsia helvetica in Danish Ixodes ricinus ticks. APMIS 115, 225-230 (2007).

24. Fraenkel, C.-J., Garpmo, U. \& Berglund, J. Determination of novel Borrelia genospecies in Swedish Ixodes ricinus ticks. J. Clin. Microbiol. 40, 3308-3312 (2002).

25. Wilhelmsson, P. et al. Prevalence, diversity, and load of Borrelia species in ticks that have fed on humans in regions of Sweden and Åland Islands, Finland with different Lyme borreliosis incidences. PLoS ONE 8, e81433 (2013). 
26. Vennestrøm, J., Egholm, H. \& Jensen, P. M. Occurrence of multiple infections with different Borrelia burgdorferi genospecies in Danish Ixodes ricinus nymphs. Parasitol. Int. 57, 32-37 (2008).

27. Kjelland, V., Stuen, S., Skarpaas, T. \& Slettan, A. Prevalence and genotypes of Borrelia burgdorferi sensu lato infection in Ixodes ricinus ticks in southern Norway. Scand. J. Infect. Dis. 42, 579-585 (2010).

28. Klitgaard, K., Kjær, L. J., Isbrand, A., Hansen, M. F. \& Bødker, R. Multiple infections in questing nymphs and adult female Ixodes ricinus ticks collected in a recreational forest in Denmark. Ticks Tick. Borne. Dis. 10, 1060-1065 (2019).

29. Maraspin, V., Ruzic-Sabljic, E. \& Strle, F. Lyme borreliosis and Borrelia spielmanii. Emerg. Infect. Dis. 12, 1177-1177 (2006).

30. Rudenko, N., Golovchenko, M., Grubhoffer, L. \& Oliver, J. H. Updates on Borrelia burgdorferi sensu lato complex with respect to public health. Ticks Tick. Borne. Dis. 2, 123-128 (2011).

31. Fertner, M. E., Mølbak, L., Pihl, T. P. B., Fomsgaard, A. \& Bødker, R. First detection of tick-borne "Candidatus Neoehrlichia mikurensis" in Denmark 2011. Eurosurveillance 17, 20096 (2012).

32. Quarsten, H. et al. Candidatus Neoehrlichia mikurensis and Borrelia burgdorferi sensu lato detected in the blood of Norwegian patients with erythema migrans. Ticks Tick. Borne. Dis. 8, 715-720 (2017).

33. Stuen, S., Granquist, E. G. \& Silaghi, C. Anaplasma phagocytophilum-a widespread multi-host pathogen with highly adaptive strategies. Front. Cell. Infect. Microbiol. 3, 31 (2013).

34. Fomsgaard, A. et al. Tick-borne encephalitis virus, Zealand, Denmark, 2011. Emerg. Infect. Dis. 19, 1171-1173 (2013).

35. Jensen, P. M. et al. Transmission differentials for multiple pathogens as inferred from their prevalence in larva, nymph and adult of Ixodes ricinus (Acari: Ixodidae). Exp. Appl. Acarol. 71, 171-182 (2017).

36. Lundkvist, Å., Wallensten, A., Vene, S. \& Hjertqvist, M. Tick-borne encephalitis increasing in Sweden, 2011. Eurosurveillance 16, 19981 (2011).

37. Svensson, J., Hunfeld, K.-P. \& Persson, K. E. M. High seroprevalence of Babesia antibodies among Borrelia burgdorferi-infected humans in Sweden. Ticks Tick. Borne. Dis. 10, 186-190 (2019).

38. Mørch, K., Holmaas, G., Frolander, P. S. \& Kristoffersen, E. K. Severe human Babesia divergens infection in Norway. Int. J. Infect. Dis. 33, 37-38 (2015).

39. Uhnoo, I. et al. First documented case of human babesiosis in Sweden. Scand. J. Infect. Dis. 24, 541-547 (2009).

40. Dumler, J. S., Barat, N. C., Barat, C. E. \& Bakken, J. S. Human granulocytic anaplasmosis and macrophage activation. Clin. Infect. Dis. 45, 199-204 (2007).

41. Nilsson, K., Elfving, K. \& Påhlson, C. Rickettsia helvetica in patient with meningitis, Sweden, 2006. Emerg. Infect. Dis. 16, 490-492 (2010).

42. Frivik, J. O., Noraas, S., Grankvist, A., Wennerås, C. \& Quarsten, H. En mann i 60-årene fra Sørlandet med intermitterende feber (In Norwegian). Tidsskr. Den Nor. legeforening 137, (2017).

43. Grankvist, A. et al. Infections with the tick-borne bacterium 'Candidatus Neoehrlichia mikurensis' mimic noninfectious conditions in patients with B cell malignancies or autoimmune diseases. Clin. Infect. Dis. 58, 1716-1722 (2014).

44. Welinder-Olsson, C., Kjellin, E., Vaht, K., Jacobsson, S. \& Wenneras, C. First case of human 'Candidatus Neoehrlichia mikurensis' infection in a febrile patient with chronic lymphocytic leukemia. J. Clin. Microbiol. 48, 1956-1959 (2010).

45. Rizzoli, A. et al. Ixodes ricinus and its transmitted pathogens in urban and peri-urban areas in Europe: new hazards and relevance for public health. Front. Public Health 2, 251 (2014).

46. Michelitsch, A., Wernike, K., Klaus, C., Dobler, G. \& Beer, M. Exploring the reservoir hosts of tick-borne encephalitis virus. Viruses vol. 11 (2019).

47. Keesing, F. et al. Reservoir competence of vertebrate hosts for Anaplasma phagocytophilum. Emerg. Infect. Dis. 18, 2013-2016 (2012).

48. Zhan, L. et al. Anaplasma phagocytophilum in livestock and small rodents. Vet. Microbiol. 144, 405-408 (2010).

49. Portillo, A., Santibáñez, P., Palomar, A. M., Santibáñez, S. \& Oteo, J. A. Candidatus Neoehrlichia mikurensis, Europe. New Microbes New Infect. 22, 30-36 (2018).

50. Jenkins, A. et al. Detection of Candidatus Neoehrlichia mikurensis in Norway up to the northern limit of Ixodes ricinus distribution using a novel real time PCR test targeting the groEL gene. BMC Microbiol. 19, 199 (2019).

51. Obiegala, A. \& Silaghi, C. Candidatus Neoehrlichia mikurensis-recent insights and future perspectives on clinical cases, vectors, and reservoirs in Europe. Curr. Clin. Microbiol. Rep. 5, 1-9 (2018).

52. Yabsley, M. J. \& Shock, B. C. Natural history of zoonotic Babesia: role of wildlife reservoirs. Int. J. Parasitol. Parasites Wildl. 2, 18-31 (2013).

53. Sprong, H. et al. Ixodes ricinus ticks are reservoir hosts for Rickettsia helvetica and potentially carry flea-borne Rickettsia species. Parasit. Vectors 2, 41 (2009).

54. Jaenson, T. G. T. et al. Risk indicators for the tick Ixodes ricinus and Borrelia burgdorferi sensu lato in Sweden. Med. Vet. Entomol. 23, 226-237 (2009).

55. Hudson, P. J. et al. Tick-borne encephalitis virus in northern Italy: molecular analysis, relationships with density and seasonal dynamics of Ixodes ricinus. Med. Vet. Entomol. 15, 304-313 (2001).

56. Nazzi, F. et al. Ticks and Lyme borreliosis in an alpine area in northeast Italy. Med. Vet. Entomol. 24, 220-226 (2010).

57. Hubalek, Z., Halouzka, J. \& Juricova, Z. Longitudinal surveillance of the tick Ixodes ricinus for Borreliae. Med. Vet. Entomol. 17, 46-51 (2003).

58. Lindström, A. \& Jaenson, T. G. T. Distribution of the common tick, Ixodes ricinus (Acari: Ixodidae), in different vegetation types in southern Sweden. J. Med. Entomol. 40, 375-378 (2003).

59. Mejlon, H. A. \& Jaenson, T. G. T. Jaenson (1993) Seasonal prevalence of Borrelia burgdorferi in Ixodes ricinus in different vegetation types in Sweden. Scand. J. Infect. Dis. 25, 449-456 (2009).

60. Tack, W. et al. Local habitat and landscape affect Ixodes ricinus tick abundances in forests on poor, sandy soils. For. Ecol. Manag. 265, 30-36 (2012)

61. Walhström, L. K. \& Kjellander, P. Ideal free distribution and natal dispersal in female roe deer. Oecologia 103, 302-308 (1995).

62. Zeman, P. Objective assessment of risk maps of tick-borne encephalitis and Lyme borreliosis based on spatial patterns of located cases. Int. J. Epidemiol. 26, 1121-1129 (1997).

63. Jat, M. K. \& Mala, S. Application of GIS and space-time scan statistic for vector born disease clustering. In ICEGOV' 17 Proceedings of the 10th International Conference on Theory and Practice of Electronic Governance (2017) https://doi.org/10.1145/30472 73.3047361 .

64. Hönig, V. et al. Model of risk of exposure to Lyme borreliosis and tick-borne encephalitis virus-infected ticks in the border area of the Czech Republic (South Bohemia) and Germany (Lower Bavaria and Upper Palatinate). Int. J. Environ. Res. Public Health 16, 1173 (2019).

65. Randolph, S. E. \& Rogers, D. J. Fragile transmission cycles of tick-borne encephalitis virus may be disrupted by predicted climate change. Proc. Biol. Sci. 267, 1741-1744 (2000).

66. Stefanoff, P. et al. A Predictive model has identified tick-borne encephalitis high-risk areas in regions where no cases were reported previously, Poland, 1999-2012. Int. J. Environ. Res. Public Health 15, 677 (2018).

67. Kjær, L. J. et al. Predicting and mapping human risk of exposure to Ixodes ricinus nymphs using climatic and environmental data, Denmark, Norway and Sweden, 2016. Eurosurveillance 24, 1800101 (2019). 
68. Kjær, L. J. et al. Predicting the spatial abundance of Ixodes ricinus ticks in southern Scandinavia using environmental and climatic data. Sci. Rep. 9, 18144 (2019).

69. Kjær, L. J. et al. Spatial data of Ixodes ricinus instar abundance and nymph pathogen prevalence, Scandinavia, 2016-2017. Collection https://doi.org/10.6084/m9.figshare.c.4938270.v1 (2020).

70. Kjær, L. J. et al. Spatial data of Ixodes ricinus instar abundance and nymph pathogen prevalence, Scandinavia, 2016-2017. Sci. Data $7,1-7$ (2020).

71. Scharlemann, J. P. W. et al. Global data for ecology and epidemiology: a novel algorithm for temporal Fourier processing MODIS data. PLoS ONE 3, el408 (2008).

72. Corine Land Cover 2006 raster data. European Environment Agency https://www.eea.europa.eu/data-and-maps/data/clc-2006raster (2010).

73. Klitgaard, K., Chriél, M., Isbrand, A., Jensen, T. K. \& Bødker, R. Identification of Dermacentor reticulatus ticks carrying Rickettsia raoultii on migrating jackal, Denmark. Emerg. Infect. Dis. 23, 2072-2074 (2017).

74. Moutailler, S. et al. Co-infection of ticks: the rule rather than the exception. PLoS Negl. Trop. Dis. 10, e0004539 (2016).

75. Reye, A. L. et al. Prevalence of tick-borne pathogens in Ixodes ricinus and Dermacentor reticulatus ticks from different geographical locations in Belarus. PLoS ONE 8, e54476 (2013).

76. R Development Core Team. R: A language and environment for statistical computing. $R$ Foundation for Statistical Computing https://www.r-project.org (2018).

77. Cowling, D. W., Gardner, I. A. \& Johnson, W. O. Comparison of methods for estimation of individual-level prevalence based on pooled samples. Prev. Vet. Med. 39, 211-225 (1999).

78. ESRI. ArcGIS Desktop: Release 10.6.1. Redlands, CA: Environmental Systems Research Institute. (2017).

79. Kulldorff M. and Information Management Services, I. SaTScan ${ }^{\mathrm{TM}}$ v9.6: Software for the spatial and space-time scan statistics www.satscan.org, 2018.

80. Kleinman, K. rsatscan: Tools, classes, and methods for interfacing with SaTScan stand-alone software. (2015).

81. Kulldorff, M. A spatial scan statistic. Communications in Statistics - Theory and Methods vol. 26 https://www.tandfonline.com/ doi/abs/10.1080/03610929708831995 (1997).

82. Han, J. et al. Using Gini coefficient to determining optimal cluster reporting sizes for spatial scan statistics. Int. J. Health Geogr. 15, 27 (2016).

83. Kuhn., M., Contributions from Jed Wing, Steve Weston, Andre Williams, Chris Keefer, Allan Engelhardt, T., Cooper, Zachary Mayer, Brenton Kenkel, the R Core Team, Michael Benesty, Reynald Lescarbeau, A. Z. \& Luca Scrucca, Yuan Tang, C. C. and T. H. caret: Classification and regression training. R package version 6.0-81. https://CRAN.R-project.org/package=caret. (2018).

84. Elith, J., Leathwick, J. R. \& Hastie, T. A working guide to boosted regression trees. J. Anim. Ecol. 77, 802-813 (2008).

85. Vapnik, V., Golowich, S. E. \& Smola, A. Support vector method for function approximation, regression estimation, and signal processing. in Advances in Neural Information Processing Systems 9 (eds. Mozer, M., Jordan, M. \& Petsche, T.) 281-287 (MIT Press., 1997).

86. Sanz, H., Valim, C., Vegas, E., Oller, J. M. \& Reverter, F. SVM-RFE: selection and visualization of the most relevant features through non-linear kernels. BMC Bioinform. 19, 432 (2018).

87. Ghojogh, B., Ca, B., Crowley, M. \& Ca, M. The theory behind overfitting, cross validation, rRegularization, bagging, and boosting: tTutorial. https://arxiv.org/abs/1905.12787 [stat.ML] 1-23 (2019).

88. Skarphédinsson, S., Jensen, P. M. \& Kristiansen, K. Survey of tickborne infections in Denmark. Emerg. Infect. Dis. 11, 1055-1061 (2005).

89. Quarsten, H., Skarpaas, T., Fajs, L., Noraas, S. \& Kjelland, V. Tick-borne bacteria in Ixodes ricinus collected in southern Norway evaluated by a commercial kit and established real-time PCR protocols. Ticks Tick. Borne. Dis. 6, 538-544 (2015).

90. Wilhelmsson, P. et al. Prevalence and diversity of Borrelia species in ticks that have bitten humans in Sweden. J. Clin. Microbiol. 48, 4169-4176 (2010).

91. Strnad, M., Hönig, V., Růžek, D., Grubhoffer, L. \& Rego, R. O. M. Europe-wWide meta-analysis of Borrelia burgdorferi sensu lato prevalence in questing Ixodes ricinus ticks. Appl. Environ. Microbiol. 83, 3838 (2017).

92. Mysterud, A. et al. Tick abundance, pathogen prevalence, and disease incidence in two contrasting regions at the northern distribution range of Europe. Parasit. Vectors 11, 309 (2018).

93. Severinsson, K., Jaenson, T. G., Pettersson, J., Falk, K. \& Nilsson, K. Detection and prevalence of Anaplasma phagocytophilum and Rickettsia helvetica in Ixodes ricinus ticks in seven study areas in Sweden. Parasit. Vectors 3, 66 (2010).

94. Karlsson, M. E. \& Andersson, M. O. Babesia species in questing Ixodes ricinus, Sweden. Ticks Tick. Borne. Dis. 7, 10-12 (2016).

95. Øines, Ø., Radzijevskaja, J., Paulauskas, A. \& Rosef, O. Prevalence and diversity of Babesia spp. in questing Ixodes ricinus ticks from Norway. Parasit. Vectors 5, 156 (2012).

96. Andersson, M., Bartkova, S., Lindestad, O. \& Råberg, L. Co-Infection with 'Candidatus Neoehrlichia mikurensis' and Borrelia afzelii in Ixodes ricinus Ticks in Southern Sweden. Vector-Borne Zoonotic Dis. 13, 438-442 (2013).

97. Pedersen, B. N. et al. Distribution of Neoehrlichia mikurensis in Ixodes ricinus ticks along the coast of Norway: the western seaboard is a low-prevalence region. Zoonoses Public Health https://doi.org/10.1111/zph.12662 (2019).

98. Kantsø, B., Bo Svendsen, C., Moestrup Jensen, P., Vennestrøm, J. \& Krogfelt, K. A. Seasonal and habitat variation in the prevalence of Rickettsia helvetica in Ixodes ricinus ticks from Denmark. Ticks Tick. Borne. Dis. 1, 101-103 (2010).

99. Solano-Gallego, L., Sainz, Á., Roura, X., Estrada-Peña, A. \& Miró, G. A review of canine babesiosis: the European perspective. Parasit. Vectors 9,336 (2016).

100. Randolph, S. E. The shifting landscape of tick-borne zoonoses: tick-borne encephalitis and Lyme borreliosis in Europe. Philos. Trans. R. Soc. Lond. B. Biol. Sci. 356, 1045-1056 (2001).

101. Sumilo, D. et al. Tick-borne encephalitis in the Baltic States : Identifying risk factors in space and time. Int. J. Med. Microbiol. 296(Suppl), 76-79 (2006).

102. Sumilo, D. et al. Socio-economic factors in the differential upsurge of tick-borne encephalitis in Central and Eastern Europe. Rev. Med. Virol. 18, 81-95 (2008).

103. Randolph, S. E., Green, R. M., Peacey, M. F. \& Rogers, D. J. Seasonal synchrony : the key to tick-borne encephalitis foci identified by satellite data. Parasitology 121, 15-23 (2000).

104. Halos, L. et al. Ecological factors characterizing the prevalence of bacterial tick-borne pathogens in Ixodes ricinus ticks in pastures and woodlands. Appl. Environ. Microbiol. 76, 4413-4420 (2010).

105. Sjörs, H. Nordisk växtgeografi (in Swedish) (Bonniers, Scandinavian University Books, 1967).

\section{Acknowledgements}

We thank Simon Friis-Wandall, Mette Frimodt Hansen, Caroline Greisen, Ana Carolina Cuellar, Najmul Haider, Leif Kristian Sortedal, Philip Thomassen Neset, Preben Ottesen, Alaka Lamsal, Ruchika Shakya, Martin Strnad, Hanne Quarsten, Sølvi Noraas, Åslaug Rudjord Lorentzen, Chiara Bertacco, Kevin Hohwald, Catharina Schmidt, Coco de Koning, and Wenche Okstad for assistance in the field. We thank the late Anastasia Isbrand for help with DNA preparation and PCR assays. We would also like to thank the Danish Ministry of the Environment, 
The Forest and Nature Agency as well as many private landowners for allowing us access to their properties to conduct our sampling.

\section{Author contributions}

L.J.K. planned and managed the fieldwork set-up, performed fieldwork in Denmark and contributed to field work in Sweden, analysed the data and drafted the manuscript. R.B. planned the original study, contributed to analysis and drafting the manuscript. K.K. performed DNA preparation and the PCR assays and contributed to drafting the manuscript. A.S., K.S.E., H.E.H.L., K.M.P., A.K.A., L.K., V.K., A.S. and S.S. organised and conducted fieldwork in Norway and contributed to drafting the manuscript. P.K., M.C. and M.T. organised and conducted to fieldwork in Sweden and contributed to drafting the manuscript. A.B. contributed to analysis and drafting the manuscript. L.M.J. contributed to fieldwork, DNA preparation and drafting the manuscript. All authors read and approved the final version of the manuscript.

\section{Competing interests}

The authors declare no competing interests.

\section{Additional information}

Supplementary information is available for this paper at https://doi.org/10.1038/s41598-020-76334-5.

Correspondence and requests for materials should be addressed to L.J.K.

Reprints and permissions information is available at www.nature.com/reprints.

Publisher's note Springer Nature remains neutral with regard to jurisdictional claims in published maps and institutional affiliations.

(c) (i) Open Access This article is licensed under a Creative Commons Attribution 4.0 International License, which permits use, sharing, adaptation, distribution and reproduction in any medium or format, as long as you give appropriate credit to the original author(s) and the source, provide a link to the Creative Commons licence, and indicate if changes were made. The images or other third party material in this article are included in the article's Creative Commons licence, unless indicated otherwise in a credit line to the material. If material is not included in the article's Creative Commons licence and your intended use is not permitted by statutory regulation or exceeds the permitted use, you will need to obtain permission directly from the copyright holder. To view a copy of this licence, visit http://creativecommons.org/licenses/by/4.0/.

(C) The Author(s) 2020 